\title{
Pedestal electron collisionality and toroidal rotation during ELM-crash suppression phase under $n=1$ RMP in KSTAR
}

Cite as: Phys. Plasmas 27, 112501 (2020); https://doi.org/10.1063/5.0024055

Submitted: 04 August 2020 . Accepted: 04 October 2020 . Published Online: 02 November 2020

(D) Minwoo Kim, (D) J. Lee, W. H. Ko, S.-H. Hahn, (D) Y. In, (D) Y. M. Jeon, W. Suttrop, (D) S. K. Kim, G. Y. Park, (D) J.-W. Juhn, and J. H. Lee

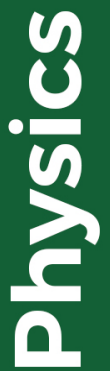

\section{ARTICLES YOU MAY BE INTERESTED IN}

The combined kinetic effects of the ion temperature gradient and the velocity shear of a plasma flow parallel to the magnetic field on the drift-Alfven instabilities

Physics of Plasmas 27, 112103 (2020); https://doi.org/10.1063/5.0021634

Excitation of the axisymmetric Alfvén eigenmodes by micro-turbulence Physics of Plasmas 27, 114503 (2020); https://doi.org/10.1063/5.0022748

The influence of boundary and edge-plasma modeling in computations of axisymmetric vertical displacement

Physics of Plasmas 27, 112505 (2020); https://doi.org/10.1063/5.0023604

\section{AlP Advances Fluids and Plasmas Collection}




\title{
Pedestal electron collisionality and toroidal rotation during ELM-crash suppression phase under $n=1$ RMP in KSTAR
}

Cite as: Phys. Plasmas 27, 112501 (2020); doi: 10.1063/5.0024055

Submitted: 4 August $2020 \cdot$ Accepted: 4 October 2020 •

Published Online: 2 November 2020

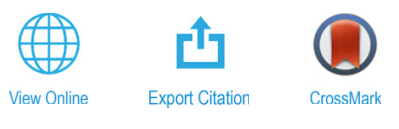

Minwoo Kim, ${ }^{1, \text { a) }}$ (D) J. Lee, ${ }^{1}$ (D) W. H. Ko, ${ }^{1}$ S.-H. Hahn, ${ }^{1}$ Y. In, ${ }^{2}$ (D) Y. M. Jeon, ${ }^{1}$ (D) W. Suttrop, ${ }^{3}$ S. K. Kim, ${ }^{4, b)}$ (DD G. Y. Park, J.-W. Juhn, ${ }^{\top}$ iD and J. H. Lee

\author{
AFFILIATIONS \\ ${ }^{7}$ National Fusion Research Institute, Daejeon 34133, South Korea \\ ${ }^{2}$ Ulsan National Institute of Science and Technology, Ulsan 44919, South Korea \\ ${ }^{3}$ Max Planck Institute for Plasma Physics, Garching 85748, Germany \\ ${ }^{4}$ Department of Energy System Engineering, Seoul National University, Seoul 08826, South Korea \\ ${ }^{a}$ Author to whom correspondence should be addressed: minwookim@nfri.re.kr \\ ${ }^{b)}$ Present address: Mechanical \& Aerospace Engineering Department, Princeton University, Princeton, NJ 08544, USA
}

\begin{abstract}
Excellent reproducibility of KSTAR resonant magnetic perturbation (RMP)-driven, edge-localized mode (ELM)-crash suppression enables us to construct a database reliably for the study of ELM-crash suppression conditions. To establish a high-fidelity database, we have selected one of the frequently used RMP configurations in KSTAR, $n=1,+90^{\circ}$ phasing RMP-coil configuration. A series of fitting curves based on edge profile diagnostics data is used for normalized electron collisionality $\left(\nu_{\mathrm{e}}^{*}\right)$ and plasma toroidal rotation $\left(V_{\text {tor }}\right)$ at pedestal top. Since ITER is expected to employ slowly rotating, low-collisionality, high-density plasmas whose conditions are not readily accessible in the existing devices, the exploration and understanding of these two parameters would be important not only for RMP-driven, ELM-crash-suppression physics but also for the success of ITER. The data points for the ELM-crash suppression phase are in the range of $0.2<\nu_{\text {e.ped }}^{*}<1.1$ with $Z_{\text {eff }}=2$ assumption and $V_{\text {tor,ped }}>40 \mathrm{~km} / \mathrm{s}$. Suppression thresholds or boundaries in $\nu_{\text {e.ped }}^{*}$ or $V_{\text {tor,ped }}$ are not confirmed in the investigated parameter ranges so far. The KSTAR database still needs additional experimental datasets in ITER-relevant conditions $\left(\nu_{\mathrm{e}, \mathrm{ped}}^{*} \sim 0.1\right.$ and low-torque low-rotation) to confirm the boundary of the ELM-crash suppression window in KSTAR and reduce the uncertainties of the RMP ELM-crash control technique in ITER. In both $\nu_{\mathrm{e} \text {,ped }}^{*}$ and $V_{\text {tor,ped }}$ parameters, the phase-space distribution of the ELM-crash suppression has no clear distinction from that of the ELM-crash mitigation. Linear discriminant analysis provides a linear combination of parameters relevant to ELM-crash suppression, best separating two data categories. Recursive feature elimination indicates that $n_{\mathrm{e}, \text { ped }}$ and $I_{\mathrm{RMP}}$, as well as $\nu_{\mathrm{e}, \mathrm{ped}}^{*}$ and $V_{\text {tor,ped }}$, are critical variables in the separation of the data groups.
\end{abstract}

Published under license by AIP Publishing. https://doi.org/10.1063/5.0024055

\section{INTRODUCTION}

The edge-localized mode (ELM), driven by the steep pressure gradient and high current density in the pedestal region of $\mathrm{H}$-mode plasmas, leads to a quasi-periodic collapse of the pedestal structure called an ELM-crash. In an ITER-size tokamak, it is anticipated that the heat and particle flux due to a single ELM-crash threatens the integrity of the first plasma-facing components (PFC). ${ }^{1}$ The application of resonant magnetic perturbation (RMP) is proposed to relieve the heat flux onto the PFC by controlling the ELM-crash. Since the first demonstration of ELM-crash suppression by the RMP technique in DIII-D experiment, ${ }^{2}$ various tokamaks have confirmed that the RMP would be an ideal tool to control the ELM-crashes effectively in either suppression or mitigation: $\mathrm{KSTAR}^{3-7}$ ASDEX Upgrade, ${ }^{8,9}$ MAST, ${ }^{10}$ and JET. ${ }^{11}$

The access conditions to ELM-crash suppression have been investigated to establish robust and reliable ELM-crash control. It is commonly observed that the edge safety factor $\left(q_{95}\right)$ should be in an optimal range to stimulate a plasma response to external magnetic perturbation while avoiding mode locking. Additionally, the edge pedestal parameters for suppression windows have been characterized, for example, normalized electron collisionality $\left(\nu_{\mathrm{e}}^{*}\right)$. In DIII-D experiments, two branches of the suppression window are observed under $n=3$ RMPs: low $\nu_{\text {e,ped }}^{*}<0.5,{ }^{12,13}$ and high $\nu_{\text {e,ped }}^{*}>0.9{ }^{14,15}$ In ASDEX Upgrade, a database built from 44 discharges of $n=2$ 
RMP-experiment indicates that the suppression is achieved in a low range of $0.15<\nu_{\text {e.ped }}^{*}<0.25$. $^{9}$ In a high collisionality plasma, $\nu_{\text {e,ped }}^{*}>1.5$; in ASDEX upgrade, ELM-crash is strongly mitigated. ${ }^{8}$ As shown in multi-device comparison, ${ }^{1,10}$ the suppression windows of type-I ELM from various tokamaks, including KSTAR, are overlapping in $\nu_{\text {e.ped }}^{*}$ and pedestal electron density (normalized by the Greenwald density) space. Since then, the KSTAR edge profile diagnostics have substantially improved, enabling us to revisit the relevant issues and analyze pedestal parameters much more rigorously.

Since ITER plasmas will be slowly rotating with an order-ofmagnitude higher density compared to a typical contemporary device, it is one of the critical issues to know whether such a low level of toroidal rotation poses a risk for the effectiveness of RMP-driven, ELM-crash control. According to DIII-D experiments with carbon tiles, plasmas cannot access the suppression window in ITER-like low-torque and low-rotation conditions with $n=3$ RMPs. ${ }^{13,16}$ On the other hand, in ASDEX Upgrade with a tungsten metal wall, the ELM-crash suppression condition under $n=2$ RMPs has no clear dependence on toroidal rotation on the pedestal top. ${ }^{9}$ Thus, the KSTAR experiments with carbon tiles can provide additional data to clarify the rotation effect on ELM-crash suppression using $n=1$ RMPs.

The KSTAR in-vessel control coil (IVCC) system for RMP consists of three rows of four window-pane-like coils in the toroidal direction, ${ }^{3,17}$ of which geometrical configuration is similar to three rows of the ITER in-vessel RMP coils. ${ }^{18}$ KSTAR has provided results to understand the physics and effects of RMP-driven ELM-crash suppression based on the high reproducibility of the suppression. ${ }^{4-7,19,20}$ In KSTAR, the RMP-driven ELM-crash suppression phase is highly sustainable, in that the longest suppression phase was stably sustained up to the time scale of wall saturation by deuterium fuel $(\sim 30 \mathrm{~s}){ }^{6}{ }^{6}$ Therefore, KSTAR provides one of the best testbeds to address the remaining uncertainties of ELM-crash control in ITER. Note that KSTAR is the only major tokamak that is equipped with an in-vessel mid-plane row of coils, a similar configuration to the ITER RMP coils. The study of access conditions for ELM-crash suppression in KSTAR contributes to understanding the feasibility of the RMP technique toward ITER conditions. In this paper, we have thoroughly examined the normalized electron collisionality $\left(\nu_{\mathrm{e}}^{*}\right)$ and the toroidal rotation velocity $\left(V_{\text {tor }}\right)$ at the pedestal, considering these two parameters as the key pedestal physics quantities related to the onset of RMP-driven, ELM-crash suppression. The database of this study consists of 28 discharges of ELM-crash control experiments with reliable pedestal profile diagnostics where all the discharge employs $n=1,+90^{\circ}$ phasing RMP and has almost the same plasma shape (high triangularity $\sim 0.6$ ). The database also includes discharges of systematic pedestaldensity-scan experiments by adjusting the level of lower-divertor deuterium-gas puff. Within the parameter space investigated so far, the database provides $\nu_{\text {e,ped }}^{*}$ and $V_{\text {tor,ped }}$ ranges of RMP ELM-crash suppression.

Section II provides an outline of the database of $n=1$ RMP ELM-crash suppression experiments in KSTAR. Section III describes the results of edge-collisionality-scan experiments, included in the database. Section IV examines the edge pedestal conditions during ELM-crash suppression. Section $V$ discusses the separation between data groups of ELM-crash mitigation and ELM-crash suppression. Finally, Sec. VI gives a summary of this paper.

\section{DATABASE OF $\boldsymbol{n}=1$ RMP ELM-CRASH CONTROL EXPERIMENTS IN KSTAR}

For a decade, the KSTAR has conducted a variety of $n=1$ RMP experiments related to the ELM-crash control and physics, resulting in the first demonstration of $n=1$ RMP ELM-crash suppression, ${ }^{3}$ the exploration of the effect of RMP coil phasing on ELM-crash control, ${ }^{5,20}$ the first observation of divertor heat flux broadening in intentionally misaligned RMP configurations, ${ }^{5,6,19}$ evidence of the interaction between RMP-driven turbulence and ELM structures, ${ }^{4}$ and direct measurement of the perpendicular flow changes at the onset of ELM-crash suppression. ${ }^{7}$ A database for RMP experiments has been constructed to summarize the results of KSTAR RMP experiments. The database discussed here does not contain all discharges under $n=1$ RMP in KSTAR. In this paper, datasets from qualified discharges, having reliable profile diagnostics data, within a specific range of experimental parameters address the relationship between edge pedestal parameters and RMP-driven ELM-crash suppression.

The selected discharges, for the study of ELM-crash suppression conditions in $\nu_{\mathrm{e}}^{*}$ and $V_{\text {tor }}$ parameter space, are obtained under nearly the same operational conditions, including the plasma shape. A total number of 28 discharges in the database are documented with an $n=1$, $+90^{\circ}$ phasing RMP configuration, ${ }^{3} B_{\mathrm{T}}\left(R_{0}\right)=1.8 \mathrm{~T}$ constant, $q_{95}$ $\sim 4.9-5.5, I_{\mathrm{p}} \sim 500-560 \mathrm{kA}, \delta \sim 0.58\left[=\left(\delta_{u}+\delta_{l}\right) / 2\right.$, with upper triangularity $\left(\delta_{u}\right) \sim 0.36 \pm 0.03$, lower triangularity $\left(\delta_{l}\right) \sim 0.79 \pm 0.13$ ], and elongation $(\kappa) \sim 1.74 \pm 0.03$. Note that the $q_{95}$ range is fully within the $q_{95}$ window for the RMP-driven, ELM-crash-suppression found in the previous study. ${ }^{6}$ Unless otherwise specified, the neutral beams are the only auxiliary heating source in most discharges $\left(P_{\mathrm{NBI}} \sim 2.8-3.7 \mathrm{MW}\right)$. Nine out of 28 discharges in the database are heated by second harmonic electron cyclotron heating $(\mathrm{ECH})$ at a power of $P_{\mathrm{ECH}}$ $\sim 0.5-1.3 \mathrm{MW}$, and a reduced level of NBI at $P_{\mathrm{NBI}} \sim 1.6-2.1 \mathrm{MW}$. For control of the divertor particle fluxes, the in-vessel helium cryogenic pumps were in full operation in all the discharges included in the database. Figure 1 shows a typical poloidal cross section of the magnetic equilibrium in one of the discharges, $\# 21574$, in the database. All the discharges are in a lower single-null (LSN) configuration, whose $\nabla B$-drift direction is pointing downward, toward the X-point. Both the toroidal magnetic field and the plasma current are in the same direction or clockwise in a bird's eye view. Here, the green lines outside the first PFC (blue line) represent the location of KSTAR IVCC for magnetic perturbation.

In this study, the Thomson scattering (TS) system ${ }^{21}$ provides $n_{\mathrm{e}}$ and $T_{\mathrm{e}}$ profiles and the charge exchange spectroscopy (CES) system ${ }^{22}$ produces $T_{\mathrm{i}}$ and $V_{\text {tor }}$ profiles of carbon impurities $\left(\mathrm{C}^{6+}\right)$ at the plasma edge. All the channels of the TS and CES systems are mapped onto the mid plane, $z=0$. The pedestal profiles are quantified using a modified hyperbolic tangent curve ${ }^{23}$ based on the averaged TS and CES data for $0.2 \mathrm{~s}$ or $\pm 0.1 \mathrm{~s}$ around each time of interest. Figure 2 shows examples of the pedestal profile analysis: KSTAR discharge \#21570, one of the cases included in the database. The pedestal profiles in the ELMy phase before RMP (orange), ELM-crash mitigation (cyan), and suppression phases (blue) are on top, middle, and bottom panels of Figs. 2(a) $-2(d)$, respectively. Markers in gray indicate data points excluded in the fitting process. The shaded region of each curve represents a 95\% confidence range of the fitting curve. Although the profile around the separatrix has non-negligible uncertainty due to unavailable diagnostics for the scrape-off layer (SOL) profile, the relative error at the 


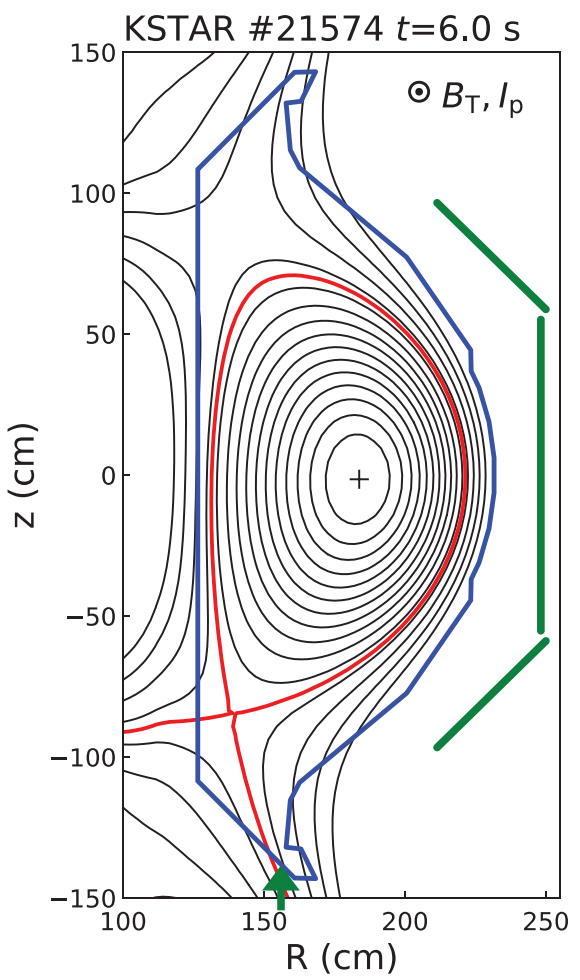

FIG. 1. Plasma equilibrium reconstruction of KSTAR discharge \#21574 at $t=6.0 \mathrm{~s}$. Red line: separatrix, blue line: first plasma-facing component surfaces, green lines: in-vessel control coils, and green arrow: divertor gas puff location.

top of the pedestal is usually less than $15 \%$. Vertical dotted lines in red represent separatrix positions from the EFIT $^{24}$ magnetic equilibrium reconstruction. Each fitting curve is shifted by $\sim 1-2 \mathrm{~cm}$ radially to match its foot position to the separatrix location from the EFIT reconstruction.

\section{EDGE COLLISIONALITY SCAN TO STUDY THE ELM-CRASH SUPPRESSION ACCESS RANGE}

The database includes seven discharges with a systematic scan of a divertor gas-puff level to vary the edge $\nu_{\mathrm{e}}^{*}$. The green arrow pointing the lower divertor region in Fig. 1 indicates the gas-puff location. In all discharges of this scan, the plasma parameters are the same except for the gas-puff level: $B_{\mathrm{T}}\left(R_{0}\right)=1.8 \mathrm{~T}$ constant, $q_{95} \sim 5.0, I_{\mathrm{p}} \sim 530 \mathrm{kA}, \delta_{u}$ $\left(\delta_{l}\right) \sim 0.35(0.85), \kappa \sim 1.72$, and $P_{\mathrm{NBI}} \sim 2.85 \mathrm{MW}$.

Figure 3 shows the time evolution of ELMy H-mode plasmas with $n=1,+90^{\circ}$ phasing RMP and three different levels of lower divertor gas-puff. The gas-injection level is adjusted in the time interval from 1 to $4 \mathrm{~s}$ where the L-H transition occurs and is subsequently reduced to zero level gradually. The RMP is excited at $4 \mathrm{~s}$. The control voltage of the Piezo valve is used to represent the gas-puff level. At $1.0 \mathrm{~V}$ for $100 \mathrm{~ms}$, the number of particles, measured around the end of the vacuum duct, is $\sim 5 \times 10^{19}$. However, the amount of gas flow to the confined plasma is not necessarily directly proportional to the injected number of particles at a low voltage, as used in this study. The gas-puff level is set to $0.3,0.6$, and $1.2 \mathrm{~V}$ from 1 to $4 \mathrm{~s}$ in \#21575, \#21574, and \#21576, respectively [yellow lines in Figs. 3(b)-3(d)]. The change in line-averaged electron density $\left(n_{\mathrm{e}, l}\right)$ measured by mm-wave interferometry ${ }^{25}$ is consistent with the divertor gas-puff level [Fig. $3(\mathrm{a})$ ]. However, $n_{\mathrm{e}, l}$ is not directly proportional to the valve voltage. Nonetheless, $n_{\mathrm{e}, l}$ is clearly reduced (also dubbed "pump-out effect") due to the magnetic perturbation. In \#21575 [Fig. 3(b), gas-puff level: $0.3 \mathrm{~V}$ ], the size of ELM-crash indicated by the $D_{\alpha}$ signal is reduced as the RMP is applied. However, the RMP application of $I_{\mathrm{RMP}}=1.7 \mathrm{kA} /$ turn mitigates the ELM-crashes. As $I_{\mathrm{RMP}}$ gradually increases after $8 \mathrm{~s}$, the ELM-crashes are being suppressed at $I_{\mathrm{RMP}} \sim 1.76 \mathrm{kA} / \mathrm{t}$ at $t \sim 8.9 \mathrm{~s}$. Then, as expected because of the high RMP field, a disruption occurs due to mode locking at $I_{\mathrm{RMP}} \sim 2.18 \mathrm{kA} / \mathrm{t}$ at $\sim 12.3 \mathrm{~s}$. In \#21574 [Fig. $3(\mathrm{c}), 0.6 \mathrm{~V}]$, ELM-crashes are successfully suppressed with a delayed response of $\sim 0.8$ s to $I_{\mathrm{RMP}}=1.7 \mathrm{kA} /$ turn (constant during the discharge). The ECH injection causes a brief period of ELM mitigation at $10 \mathrm{~s}$ for $0.2 \mathrm{~s}$. As expected, the plasma returns to ELMy H-mode, right after the RMP is turned off at $t \sim 14.5 \mathrm{~s}$. In \#21576 [Fig. 3(d), $1.2 \mathrm{~V}$ ], RMP application with $I_{\mathrm{RMP}}=1.7 \mathrm{kA} / \mathrm{t}$ mitigates the ELM-crashes. During the early phase of mitigation, multiple small ELM crashes appear between the mitigated crashes. Increasing $I_{\mathrm{RMP}}$ after $8 \mathrm{~s}$, the ELM-crash suppression is achieved at $I_{\mathrm{RMP}} \sim 1.75 \mathrm{kA} / \mathrm{t}$ at $\sim 8.7 \mathrm{~s}$ and then sustained until $I_{\mathrm{RMP}}=2.5 \mathrm{kA} / \mathrm{t}$ without encountering mode locking. Again, right after the RMP phase, ELMy H-mode reappears, as shown in the latter part of $\# 21574$. Figure $3(\mathrm{e})$ compares the time evolution of normalized beta $\left(\beta_{\mathrm{N}}\right)$ of each discharge. In all the discharges, a $\beta_{\mathrm{N}}$ drop accompanies the density pump-out due to the RMP [Figs. 3 (a) and 3(e)]. During the RMP phase, $\beta_{\mathrm{N}}$ is $\sim 1.8-2.2$. The $\beta_{\mathrm{N}}$ value decreases further by $\sim 0.1$ after $8 \mathrm{~s}$ in \#21576 [Fig. 3(e)], which may be mainly attributable to the enhanced particle transport associated with the increased level of RMP rather than a gradual decrease in the gasinjection level. This conjecture is based on the observed density pumpout, where the increase in $I_{\mathrm{RMP}}$ accelerates the continuous reduction in $n_{\mathrm{e}, l}$. Vertical lines in Figs. 3(b)-3(d) indicate various times of interest for which pedestal parameters are fully tabulated in the database.

Figures 4(a)-4(c) show the dependence of pedestal parameters, $n_{\mathrm{e}}, T_{\mathrm{e}}$, and normalized electron collisionality $\nu_{\mathrm{e}}^{*}$, on the divertor gaspuff level, respectively. Note that all data points shown in Fig. 4 are included in the pedestal parameter database discussed in Secs. IV-V. Here, the $n_{\mathrm{e}}$ value is obtained at the position of $T_{\mathrm{e}}$ pedestal top, and $\nu_{\mathrm{e}}^{*}$ is calculated using the following equation: ${ }^{26}$

$$
\nu_{\mathrm{e}}^{*}=6.921 \times 10^{-18} \frac{R q_{95} n_{\mathrm{e}} Z_{\mathrm{eff}} \ln \Lambda_{\mathrm{e}}}{\varepsilon^{3 / 2} T_{\mathrm{e}}^{2}},
$$

where $R, Z_{\text {eff }}$, and $\varepsilon$ are the major radius, the effective ion charge, and the inverse aspect ratio, respectively, and $\ln \Lambda_{\mathrm{e}}$ $\left[=31.3-\ln \left(\sqrt{n_{\mathrm{e}}} / T_{\mathrm{e}}\right)\right]$ is the Coulomb logarithm. Unless otherwise specified in this paper, $Z_{\text {eff }}=2$ is assumed. The relative uncertainty of the obtained values, including measurement error, is usually less than $\sim 15 \%$. Note that the data points of the ELMy phase have been selected before the $n=1 \mathrm{RMP}$ is applied (i.e., $t \sim 3.5 \mathrm{~s}$ ). As the divertor gaspuff level increases, the $n_{\mathrm{e} \text {,ped }}$ value increases [Fig. 4(a)], while the $T_{\text {e,ped }}$ value decreases [Fig. 4(b)]. As a result, $\nu_{\text {e,ped }}^{*}$ increases as the amount of divertor gas-puffing increases [Fig. 4(c)]. Figures 3 and 4 show the trend of the RMP-driven ELM-crash suppression in the newly constructed database, depending on $\nu_{\mathrm{e}, \mathrm{ped}}^{*}$, for example, a $\nu_{\mathrm{e}, \mathrm{ped}}^{*}$ range in the suppression phase. Section IV describes the relationship between $\nu_{\text {e,ped }}^{*}$ and ELM-crash suppression shown in the database in detail. 
(a)

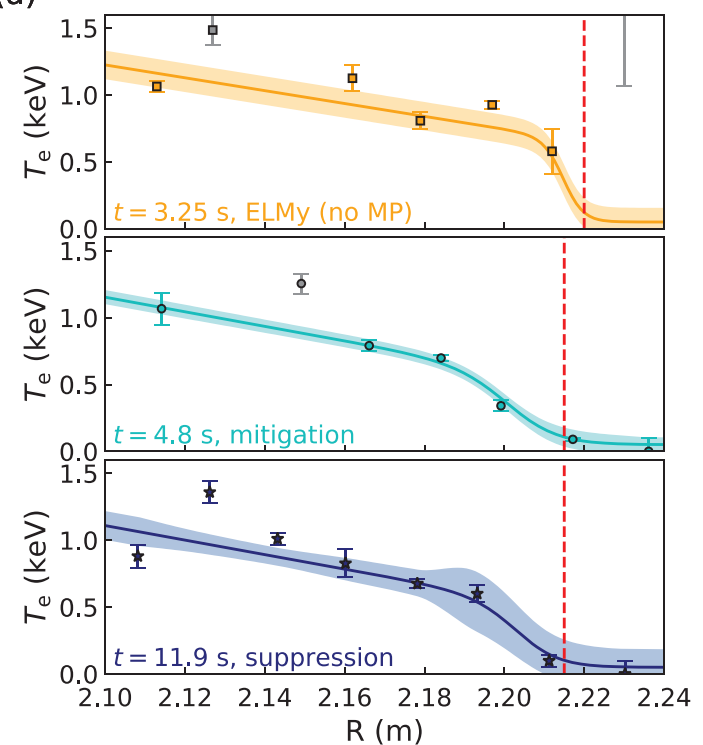

(c)

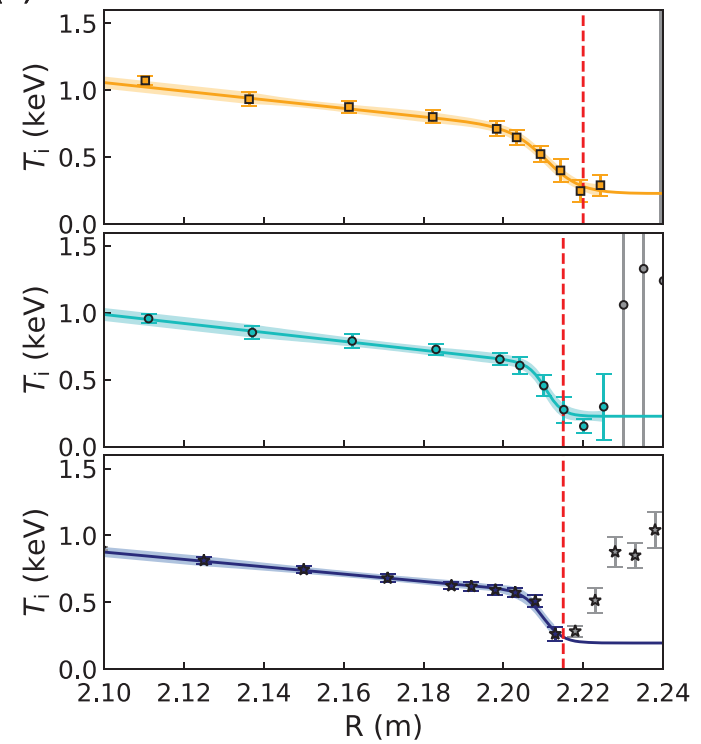

(b)

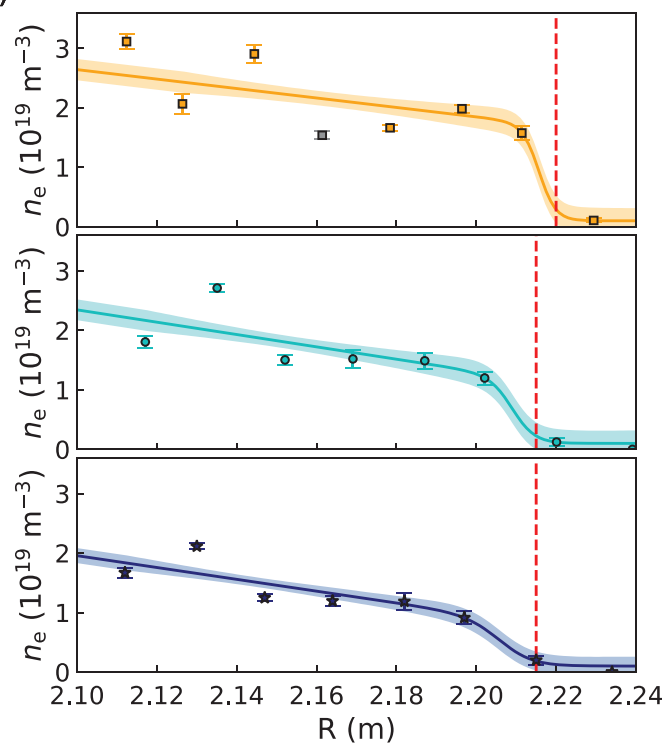

(d)

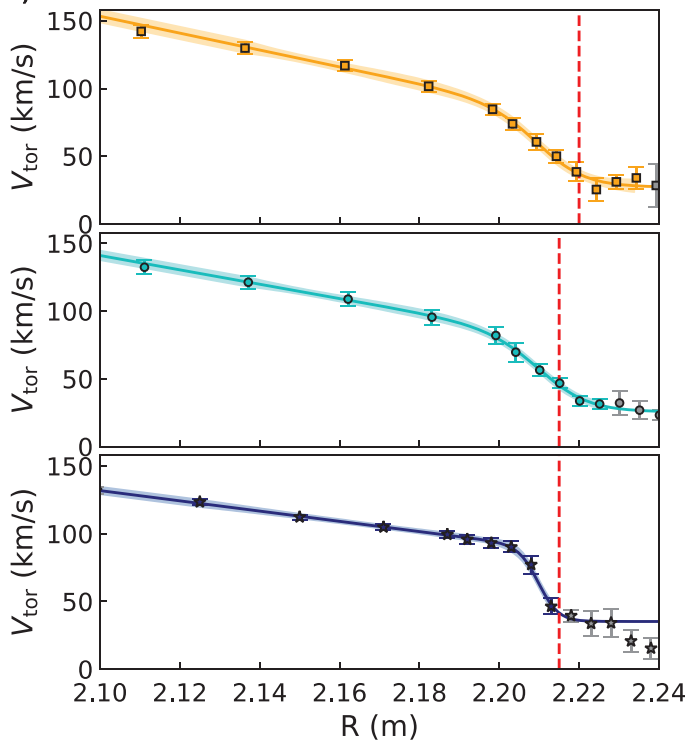

FIG. 2. Pedestal profiles of KSTAR discharge \#21570 using a modified hyperbolic tangent curve. (a) Electron temperature $\left(T_{\mathrm{e}}\right)$ and $(\mathrm{b})$ electron density $\left(n_{\mathrm{e}}\right)$ from the Thomson scattering system and $(c)$ ion temperature $\left(T_{i}\right)$ and $(d)$ toroidal rotation speed $\left(V_{\text {tor }}\right)$ of carbon impurities $\left(C^{6+}\right)$ from charge exchange spectroscopy. Top panel (orange): ELMy phase before RMP. Middle panel (cyan): mitigation phase. Bottom panel (blue): suppression phase. Shaded region: $95 \%$ confidence range of the fitting curve. Vertical dotted lines (red): separatrix position from magnetic equilibrium reconstruction. Markers in gray indicate outliers.

\section{PEDESTAL CONDITIONS DURING ELM-CRASH SUPPRESSION}

\section{A. Suppression conditions in $\nu_{\mathbf{e}}^{*}$ and $p_{\mathrm{e}}$}

Figure 5 shows the pedestal parameters evaluated at a pedestal top: $n_{\mathrm{e}, \text { ped }}$ vs $\nu_{\mathrm{e}, \text { ped }}^{*}\left[\right.$ Fig. 5(a)], $n_{\mathrm{e} \text {,ped }}$ vs $T_{\mathrm{e} \text {,ped }}$ [Fig. 5(b)], and $\nu_{\mathrm{e}, \text { ped }}^{*}$ vs $I_{\mathrm{RMP}}$ [Fig. 5(c)] space. The dataset has been categorized into three groups: the ELMy phase without RMP (square), the RMP-driven ELM-crash mitigation phase (circle), and the RMP-driven ELM-crash suppression phase (pentagram). Specific data points from $\nu_{\mathrm{e}}^{*}$ scan experiments, described in Sec. III, are indicated by the thick edge line with the same color convention as in Fig. 4. The data points of ELMmitigation and ELM-suppression phases are distributed in lower $n_{\mathrm{e} \text {,ped }}$ [Fig. 5(a)] and pe.ped [Fig. 5(b)] space compared to those of the ELMy phase. Most of the ELM-crash suppression cases are observed in $0.2<\nu_{\text {e,ped }}^{*}<1.1$, and $0.7<p_{\text {e,ped }}<1.9 \mathrm{kPa}$. The $\nu_{\mathrm{e} \text {,ped }}^{*}$ range of the suppression confirmed in the KSTAR database is partly overlapped 

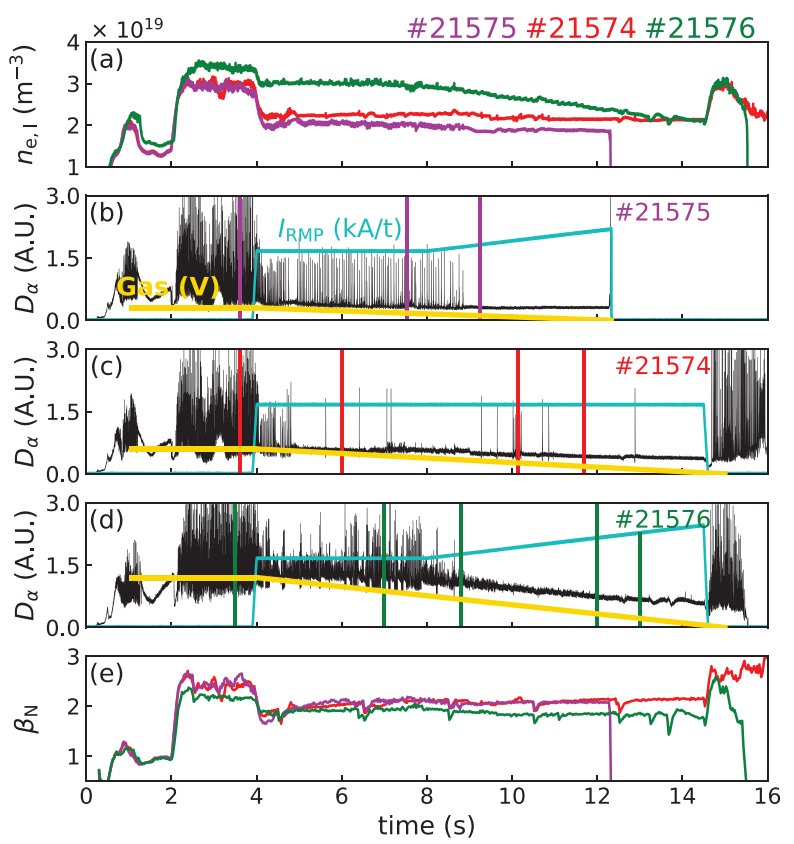

FIG. 3. Divertor gas-puff scan experiments under $n=1,+90^{\circ}$ phasing RMP. (a) Lineaveraged electron density from mm-wave interferometry. $D_{\alpha}$ and $l_{\text {RMP }}$ time traces of (b) \#21575, (c) \#21574, and (d) \#21576. Horizontal yellow lines in (b)-(d) indicate a Piezo valve voltage used to represent a low-divertor gas-puff level. (e) Plasma performance $\beta_{\mathrm{N}}$. Vertical lines in (b)-(d) indicate the time of pedestal profile analysis.

with that of other tokamaks, such as ASDEX upgrade [0.15 $<\nu_{\text {e,ped }}^{*}<0.25$ (Ref. 9)] and DIII-D [ $\nu_{\text {e,ped }}^{*}<0.5$ (Refs. 12 and 13) and $\nu_{\text {e,ped }}^{*}>0.9$ (Refs. 14 and 15)]. Note that helium is the main ion species of DIII-D $\nu_{\mathrm{e}, \text { ped }}^{*}>0.9$ plasmas. Recent nonlinear two-fluid MHD simulation using TM1 ${ }^{27,28}$ suggests that the reduced $\left|\omega_{\perp, e}\right|$ in an edge region of high rotation plasma can allow achieving the ELM-crash suppression in high collisionality (or density) conditions. This TM1 analysis can explain the ELM-crash suppression at the relatively high $\nu_{\mathrm{e}}^{*}$ pedestal of the KSTAR database, but a rigorous verification remains to be done.

In Fig. 5(a), two groups of $\nu_{\text {e,ped }}^{*}$ data points, ELM-crash mitigation and ELM-crash suppression, are not separated well in their parameter space distribution. In the cases shown in Figs. 3 and 4, there is no dramatic change in $\nu_{\mathrm{e}, \text { ped }}^{*}$ before and after the onset of ELM-crash suppression. $\nu_{\mathrm{e}}^{*}$ is subject to various edge plasma parameters, such as $q_{95}, n_{\mathrm{e}}, T_{\mathrm{e}}$, and $Z_{\text {eff }}$ as shown in Eq. (1). Therefore, the relationship between collisionality and the ELM-crash suppression condition may not be straightforward but is expected to provide the relevant critical plasma conditions at the edge pedestal. In Fig. 5(c), as $I_{\mathrm{RMP}}$ increases, ELM-crash suppression occurs in a wider $\nu_{\text {e,ped }}^{*}$ range. The suppression points are in the range of $0.35<\nu_{\mathrm{e}, \text { ped }}^{*}<0.85$ at $I_{\mathrm{RMP}} \sim 1.75 \mathrm{kA} / \mathrm{t}$ and $0.2<\nu_{\mathrm{e}, \text { ped }}^{*}<1.1$ at $I_{\mathrm{RMP}} \sim 2.0 \mathrm{kA} / \mathrm{t}$. ELM-crash suppression in \#21575 $\left[\nu_{\mathrm{e}, \mathrm{ped}}^{*} \sim 0.25\right.$, Fig. 3(b)] and \#21576 [ $\nu_{\mathrm{e} \text {,ped }}^{*} \sim 0.75$, Fig. 3(d)] is a good example of this trend; the suppression is obtained at a higher $I_{\mathrm{RMP}}$ $(>1.7 \mathrm{kA} / \mathrm{t})$ compared to \#21574 $\left[\nu_{\mathrm{e}, \text { ped }}^{*} \sim 0.45,1.7 \mathrm{kA} / \mathrm{t}\right.$, Fig. 3(c) ].

For a cross-check of the pedestal parameter range for the above ELM-crash suppression, it is compared with the previous KSTAR analysis results in Refs. 1 and 10. The previous study estimated $n_{\text {e,ped }} / n_{\mathrm{GW}}$, where $n_{\mathrm{GW}}$ is the Greenwald density limit, ${ }^{29}$ based on the line-averaged density with a scaling factor of $\sim 0.6-0.7$ because the profile diagnostics had been in commissioning. For the direct comparison of two independent datasets, $n_{\mathrm{e}, l} / n_{\mathrm{GW}}$ is appropriate rather than $n_{\text {e,ped }} / n_{\mathrm{GW}}$. The dataset for the ELM-crash suppression obtained after the improvements of edge profile diagnostics is in the range of $0.22<n_{\mathrm{e}, l} / n_{\mathrm{GW}}<0.36$, similar to the suppression range of the previous analysis $\left(0.35<n_{\mathrm{e}, l} / n_{\mathrm{GW}}<0.40\right)$ but lower. The in-vessel helium cryogenic pump is in operation regularly in the discharges discussed in this paper, which may make a difference in the density range of two suppression datasets. The $\nu_{\text {e.ped }}^{*}$ range of the suppression, $0.2<\nu_{\text {e.ped }}^{*}<1.1$, is wider compared to the previous one $\left(0.4<\nu_{\mathrm{e}, \text { ped }}^{*}<1.0\right)$, even considering the uncertainty of $n_{\mathrm{e}, \mathrm{ped}}$ in the previous analysis.

In Fig. 5(a), the majority of data points of ELM-crash suppression are below $n_{\mathrm{e} \text {,ped }}=1.5 \times 10^{19} \mathrm{~m}^{-3}$ (or $n_{\mathrm{e} \text {,ped }} / n_{\mathrm{GW}} \sim 0.2$ ). This is similar to the ASDEX Upgrade dataset, where there are no ELM-crash suppression cases above $n_{\mathrm{e} \text {,ped }}=3.3 \times 10^{19} \mathrm{~m}^{-3}\left(n_{\mathrm{e} \text {,ped }} / n_{\mathrm{GW}} \sim 0.27\right)$. However, the density threshold of the suppression in KSTAR cannot be determined yet. KSTAR can access the H-mode in a wide range of densities in auxiliary heating power usually used in RMP experiments, $P_{\text {heat }}>2.8 \mathrm{MW} .{ }^{30}$ Sufficient datasets for high density region during the RMP phase, in addition to two suppression points in $n_{\mathrm{e} \text {,ped }}>2.0 \times 10^{19} \mathrm{~m}^{-3}$ (or, $n_{\mathrm{e}, \mathrm{ped}} / n_{\mathrm{GW}} \sim 0.3$ ), are required to address whether the density threshold of ELM-crash suppression exists in KSTAR.

Although all the suppression points are below $\sim 2.5 \mathrm{kPa}$ [Fig. 5(b)], this does not guarantee the upper pressure limit of the ELMcrash suppression in KSTAR until the high-pressure region above the current datasets during the RMP phase is investigated. Additional experiments after an upgrade of total auxiliary heating power are required to verify the upper limit of pedestal pressure in the ELMcrash suppression. The lower bound of data distribution in the pressure $(\sim 0.7 \mathrm{kPa})$ is due to experimental accessibility rather than the lower pressure limit of the suppression. The fueling by neutral beams in the database discharges gets in the way of accessing the lowpressure region. In low heating power for low pressure, KSTAR has difficulties in accessing $\mathrm{H}$-mode.

\section{B. Edge rotation in ELM-crash suppression}

Figure 6 shows the toroidal rotation of plasma at the pedestal top ( $\left.V_{\text {tor,ped }}\right)$ before (squares: ELMy H-mode) and during the RMP phase (circles: ELM-crash mitigation and pentagrams: ELM-crash suppression) against $n_{\text {e,ped }}$ [Fig. 6(a)], $\nu_{\text {e.ped }}^{*}$ [Fig. 6(b)], and $I_{\text {RMP }}$ [Fig. 6(c)]. The rotation profiles are obtained in the same way as $n_{\mathrm{e}}$ and $T_{\mathrm{e}}$ profiles described in Secs. II and III. Note that in some cases of the database (e.g., ELM-crash suppression at $n_{\text {e,ped }} \sim 2.5 \times 10^{19} \mathrm{~m}^{-3}$ ), the rotation data points are missing due to an issue in the CES measurement. Based on the data distribution in $I_{\mathrm{RMP}}$ space, the data during the RMP phase are divided into two groups: low $I_{\mathrm{RMP}}$ group $\left(I_{\mathrm{RMP}}<1.9 \mathrm{kA} / \mathrm{t}\right)$ and high $I_{\mathrm{RMP}}$ group $\left(I_{\mathrm{RMP}}>1.9 \mathrm{kA} / \mathrm{t}\right)$. In Fig. 6, non-filled markers indicate the data points of the high $I_{\mathrm{RMP}}$ group.

In Figs. 6(a) and 6(b), the RMP-induced magnetic braking lowers the plasma rotation during the RMP phase, as neoclassical toroidal viscosity (NTV) torque prevails. ${ }^{31}$ Interestingly, in the ELM-crash suppression phase of both low and high $I_{\mathrm{RMP}}$ groups, $V_{\text {tor,ped }}$ has no clear dependence on $n_{\mathrm{e}, \text { ped }}$ and $\nu_{\mathrm{e} \text {,ped }}^{*}$ in the scanned parameter range. However, $V_{\text {tor,ped }}$ tends to decrease as $I_{\mathrm{RMP}}$ increases in the dataset of 

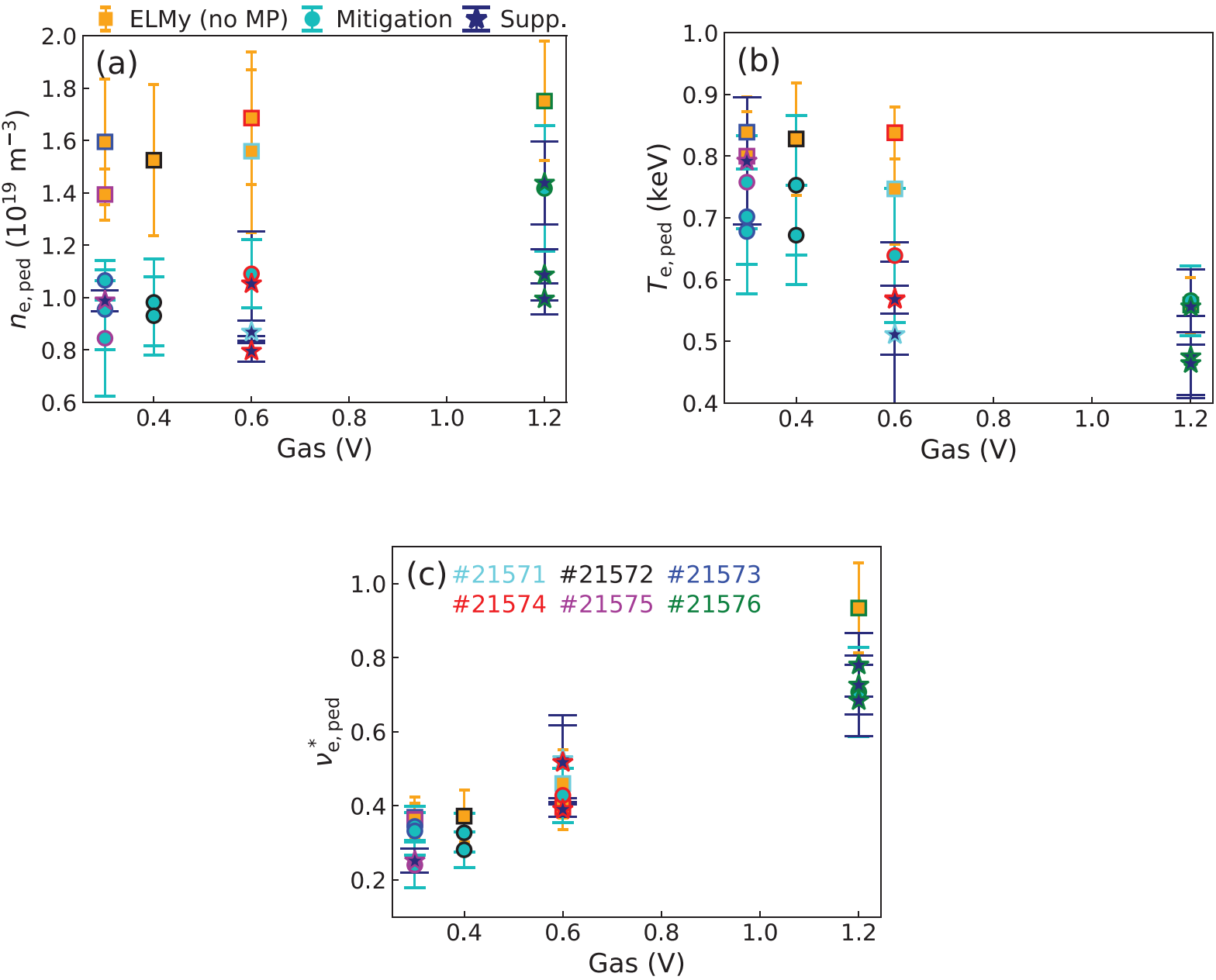

FIG. 4. Pedestal parameters according to a voltage of the Piezo valve on the lower divertor, which is a proxy for the gas-puff level. Valve voltage vs (a) $n_{\mathrm{e}}$, (b) $T_{\mathrm{e}}$, and (c) $\nu_{\mathrm{e}}^{*}$ on $T_{\mathrm{e}}$ pedestal top. Square: from the ELMy phase before RMP, circle: from the ELM-crash mitigation phase during RMP, and pentagram: from the suppression phase. The edge color of markers distinguishes each discharge data.

ELM-crash suppression [Fig. 6(c)]. In the low $I_{\mathrm{RMP}}$ group, most of the suppression points are observed at $V_{\text {tor,ped }}>70 \mathrm{~km} / \mathrm{s}$, and only mitigation cases appear below $V_{\text {tor,ped }} \sim 60 \mathrm{~km} / \mathrm{s}$. In the high $I_{\mathrm{RMP}}$ group, the suppression points are in the lower $V_{\text {tor,ped }}$ range, $40<V_{\text {tor,ped }}<70 \mathrm{~km} /$ s, compared to the low $I_{\mathrm{RMP}}$ group.

Given the dataset so far, all the ELM-crash suppression points are observed at $V_{\text {tor,ped }}>40 \mathrm{~km} / \mathrm{s}$, similar to the results from lowtorque low-rotation experiments in DIII-D describing rotation threshold. ${ }^{13,16}$ However, it does not necessarily mean that such data distribution verifies the rotation threshold for ELM-crash suppression, in that no discharges in the KSTAR database were explored yet in low-torque plasmas that may end up with the $V_{\text {tor,ped }}<40 \mathrm{~km} / \mathrm{s}$ region. Once an upgrade of $\mathrm{ECH}$ power is made in the near future, the KSTAR is expected to be capable of accessing the $V_{\text {tor,ped }}<40 \mathrm{~km} / \mathrm{s}$ region, addressing the rotation threshold issue. Alternatively, the use of nonresonant magnetic perturbation (NRMP) on top of $\mathrm{RMP}^{31}$ is being considered, as well. Another issue is that in the $V_{\text {tor,ped }}$ and $I_{\mathrm{RMP}}$ space, two data distributions of ELM-crash suppression and mitigation overlap a lot, as in $\nu_{\text {e,ped }}^{*}$ space. Section $V$ discusses conditions separating suppression from mitigation.

According to 14 out of 28 discharges in the database at the same neutral beam power $\left(P_{\mathrm{NBI}} \sim 2.85 \mathrm{MW}\right)$, the edge rotation at the pedestal top is in the range of $75<V_{\text {tor,ped }}<150 \mathrm{~km} / \mathrm{s}$ without RMP, $50<V_{\text {tor,ped }}<90 \mathrm{~km} / \mathrm{s}$ in low $I_{\mathrm{RMP}}$ cases $\left(I_{\mathrm{RMP}}<1.9 \mathrm{kA} / \mathrm{t}\right)$, and $40<V_{\text {tor,ped }}<70 \mathrm{~km} / \mathrm{s}$ in high $I_{\mathrm{RMP}}$ cases $\left(I_{\mathrm{RMP}}>1.9 \mathrm{kA} / \mathrm{t}\right)$. This suggests that the edge rotation is not a simple function of any single variable, such as an external torque or $I_{\mathrm{RMP}}$. The set of $\nu_{\mathrm{e}}^{*}$ scan experiments, described in Sec. III and Fig. 3, is revisited to discuss the edge rotation in RMP ELM-crash control experiments. Figure 7 shows the time evolution of $V_{\text {tor.ped }}$ at different lower divertor gas-puff levels. The dotted lines indicate time traces of a single CES channel around the pedestal top. Since the RMP application can cause changes in the pedestal structure, plasma size, and position, the CES channel most close to the pedestal top can be different before and during the RMP phase. In Fig. 7, two CES channels are used to show the time evolution of $V_{\text {tor,ped }}$ : channels on $R \sim 219 \mathrm{~cm}$ (before the RMP phase) and 
(a)

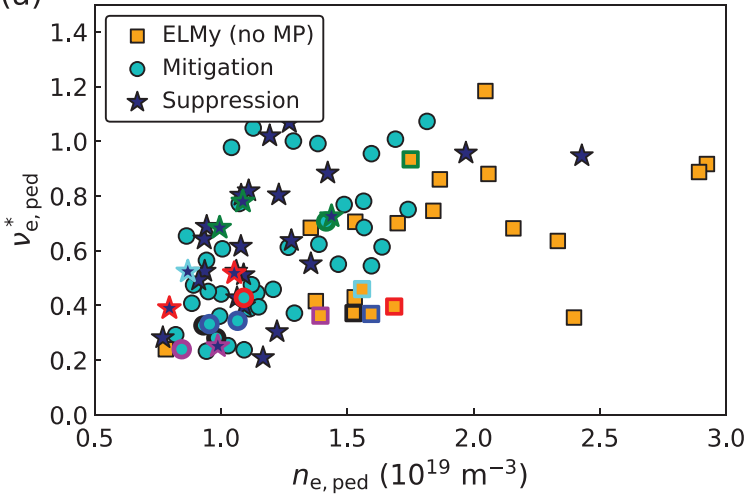

(b)

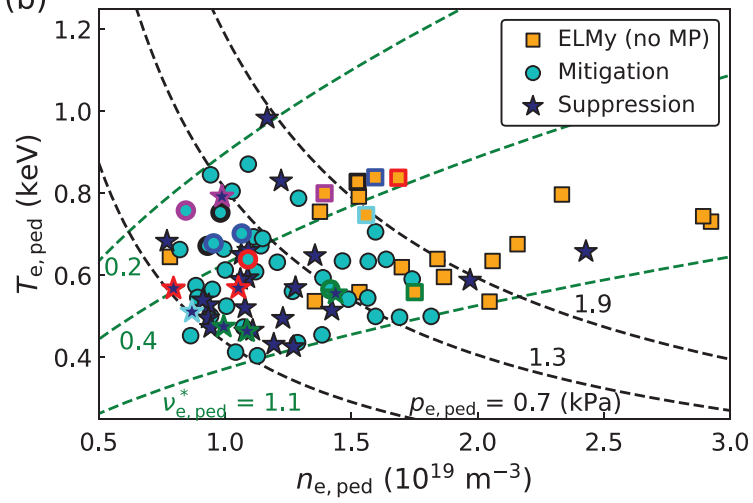

(c)

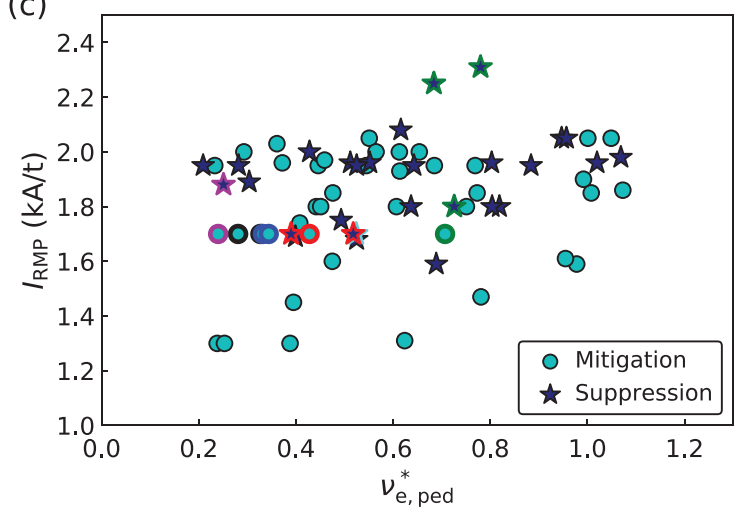

FIG. 5. (a) Normalized electron collisionality vs electron density, (b) electron temperature vs electron density, and (c) RMP current vs normalized electron collisionality. All the pedestal parameters are from the $T_{\mathrm{e}}$ pedestal top. Square: ELMy phase before RMP, circle: ELM-crash mitigation phase during RMP, and pentagram: suppression phase. The markers enclosed by a thick line represent the data points from $\nu_{\mathrm{e}}^{*}$ scan experiments, where the color convention is the same as in Fig. 4. In the $\nu_{\mathrm{e}}^{*}$ contour of (b), $R=1.85$ $\mathrm{m}, q_{95}=5.0, Z_{\text {eff }}=2.0$, and $\varepsilon=0.255$. In (c), there are only mitigation and suppression points.

$R \sim 220 \mathrm{~cm}$ (during the RMP phase). The markers represent the values at the pedestal top position of the fitting curves. The edge pedestal parameters and $I_{\mathrm{RMP}}$ values, corresponding to the markers in Fig. 7, can be found in Figs. 4-6, where the same marker convention is used. Three discharges, \#21574, \#21575, and \#21576, in Fig. 7 have the same power of neutral beams. However, even with no RMP, the plasma rotation on the pedestal top varies, depending on the levels of $n_{\mathrm{e}, l}$ and $n_{\text {e.ped }}$. As the RMP applies, the edge rotation decreases by the RMP-driven magnetic braking. $V_{\text {tor,ped }}$ becomes $\sim 80 \mathrm{~km} / \mathrm{s}$ in \#21574 and \#21575 but appears as low as $\sim 65 \mathrm{~km} / \mathrm{s}$ in \#21576. In the early phase of ELM-crash suppression after the transition from the mitigation phase, $V_{\text {tor,ped }}$ decreases further to $\sim 70 \mathrm{~km} / \mathrm{s}$ in $\# 21575\left[\nu_{\text {e.ped }}^{*} \sim 0.25\right.$, Fig. $\left.7(\mathrm{a})\right]$. Instead, $V_{\text {tor,ped }}$ increases to $\sim 80 \mathrm{~km} / \mathrm{s}$ in $\# 21576\left[\nu_{\text {e.ped }}^{*} \sim 0.75\right.$, Fig. $\left.7(\mathrm{c})\right]$. As $I_{\mathrm{RMP}}$ increases in $\# 21575$ and \#21576, $V_{\text {tor,ped }}$ decreases further during the suppression phase. The $V_{\text {tor,ped }}$ reduction rate is larger in \#21575 of low $\nu_{\text {e,ped }}^{*}$ plasma in the database, compared to $\# 21576$ of relatively high $\nu_{\text {e.ped }}^{*}$ plasma. This simple analysis on $V_{\text {tor }}$ suggests that a comprehensive approach, including density, collisionality, external torque, and (non-)magnetic torque, is necessary to estimate the plasma rotation and investigate the rotation effect on the RMPdriven ELM-crash suppression.

\section{DISCUSSION: SEPARATING THE ELM-CRASH SUPPRESSION DATA GROUP FROM THE MITIGATION DATA GROUP}

As mentioned earlier, in the qualified discharges for the KSTAR RMP experiment database, the edge pedestal conditions during the ELM-crash suppression are characterized and mapped based on the relevant equilibrium profiles. In the investigated parameter space, the distribution of ELM-crash mitigation is not entirely distinct from that of the ELM-crash suppression (Fig. 5), and $V_{\text {tor,ped }}$ alone cannot be sufficient to determine whether the ELM-crash is mitigated or suppressed (Fig. 6). This section discusses the issues of separating two data categories to clarify the ELM-crash-suppression accessible conditions.

As a basic approach, MISHKA1 ${ }^{32}$ calculates a set of ideal linear stabilities in \#21574, \#21575, and \#21576 (Figs. 3 and 7) pedestal regions, based on the fitted edge pedestal profiles. Figure 8 shows the linear stability diagrams, where $\alpha_{\max }$ and $\left\langle j_{\phi \text {,max }}\right\rangle$ represent the 
(a)

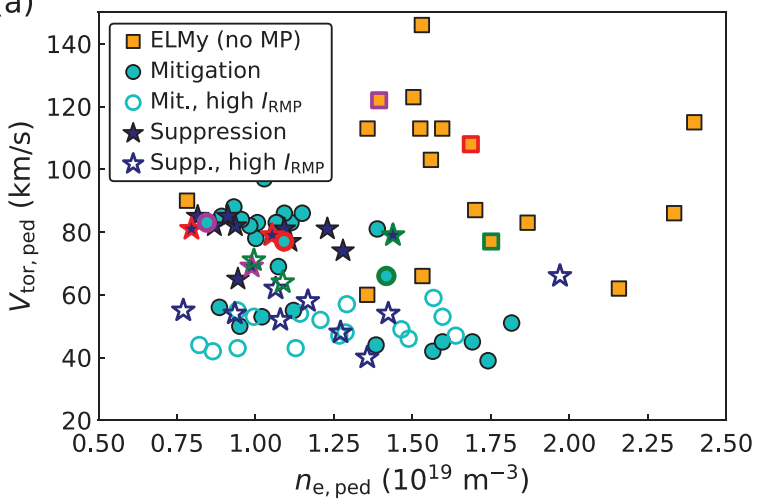

(b)

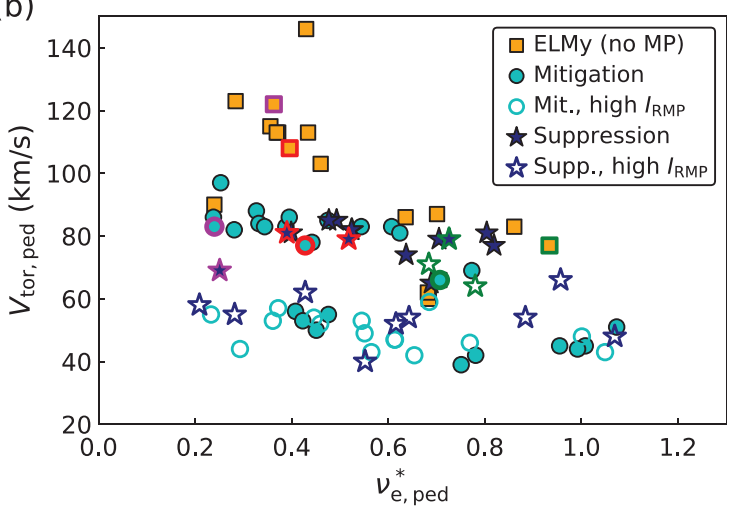

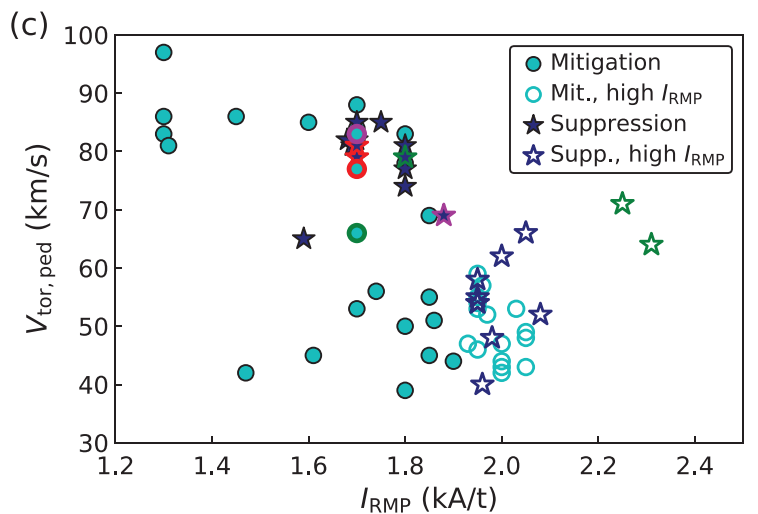

FIG. 6. Toroidal rotation velocity vs (a) electron density, (b) normalized electron collisionality, and (c) RMP current. Square: ELMy phase before RMP, circle: ELM-crash mitigation phase, and pentagram: suppression phase. Not-filled markers: high $I_{\text {RMP }}$ data group ( $\left.I_{\text {RMP }}>1.9 \mathrm{kA} / \mathrm{t}\right)$. Filled markers: low $I_{\text {RMP }}$ data group ( $\left.I_{\text {RMP }}<1.9 \mathrm{kA} / \mathrm{t}\right)$. The markers enclosed by a thick line are from \#21574 (red), \#21575 (magenta), and \#21576 (green) of collisionality scan experiments. In (c), there are only mitigation and suppression points.

maximum normalized pressure gradient ${ }^{33}$ and the maximum flux-surface-averaged toroidal current density in the pedestal region, respectively. As usual, the stability criterion is $\gamma>\omega_{*, \mathrm{i}} / 2,{ }^{34}$ where $\gamma$ is the ideal-MHD growth rate and $\omega_{*, \mathrm{i}}$ is the ion diamagnetic drift frequency. In all the cases shown in Fig. 8, the measured operation point of the ELMy phase (square) is in the unstable region, and the points of ELMcrash mitigation (circle) and ELM-crash suppression phases (pentagram) are located far away from the stability boundaries (cyan and blue solid lines in Fig. 8, respectively). Even considering error in $\alpha_{\max }$ and $\left\langle j_{\phi, \text { max }}\right\rangle$ due to uncertainty in the profile measurement around the separatrix, the operation points of the mitigation phase, where reduced but frequent ELM-crashes occur, are still in the stability region. Such a contradiction may be resolved by a recent RMP study considering three-dimensional geometry, where a local distortion of RMP-induced magnetic shear can drive highly localized ballooning modes. ${ }^{35,36}$ However, the localized destabilization is not sufficient to explain the bifurcated onset of the ELM-crash suppression phase entirely. In the KSTAR database, the $I_{\mathrm{RMP}}$ conditions and the equilibrium profiles in the early phase of ELM-crash suppression are not significantly different from those of the mitigation phase right before transition.
Since the ideal linear stability analysis alone is not enough to differentiate ELM-crash suppression from mitigation, linear discriminant analysis (LDA) $)^{37-39}$ is introduced to separate the two data categories. The goal of LDA is to find a projection direction separating data categories (so-called class) using a linear combination of variables (so-called features). The best projection vector $(w)$ maximizes the distance between classes $\left[w^{T} S_{B} w\right.$, where $S_{B}=\Sigma_{i}^{L} N_{i} / N\left(m_{i}-m_{0}\right)\left(m_{i}-m_{0}\right)^{T}$ : betweenclass scatter matrix] and minimizes the variance of data points within a class $\left[w^{T} S_{W} w\right.$, where $S_{W}=\Sigma_{i}^{L} \Sigma_{j}^{N_{i}} 1 / N\left(x_{j}^{(i)}-m_{i}\right)\left(x_{j}^{(i)}-m_{i}\right)^{T}$ : within-class scatter matrix]. Here, $L$ is the number of classes, $N$ is the total number of data points, $N_{i}$ is the number of data points within the $i$-th class, $m_{i}$ is the mean vector for the $i$-th class, and $m_{0}$ is the mean vector for entire data points. The solution vectors can be found by maximizing the Rayleigh quotient $\left[J(w)=w^{T} S_{B} w / w^{T} S_{W} w\right] .^{37}$ This process is equivalent to the eigenvalue problem of the $S_{W}^{-1} S_{B}$ matrix $\left[S_{W}^{-1} S_{B} w=\lambda w\right.$, where $\left.\lambda=J(w)\right]$, which has at most $L-1$ non-zero eigenvalues. ${ }^{38}$ The eigenvector corresponding to the maximum eigenvalue projects data points onto the new axis maximizing separation between classes.

In the RMP-driven ELM-crash control issue, seven variables, i.e., $n_{\mathrm{e} \text {,ped }}$ (in $10^{19} \mathrm{~m}^{-3}$ ), $T_{\mathrm{e} \text {,ped }}$ (in $\mathrm{eV}$ ), $T_{\mathrm{i} \text {,ped }}$ (in $\mathrm{eV}$ ), $V_{\text {tor,ped }}($ in $\mathrm{km} / \mathrm{s}$ ), 
(a) \#21575

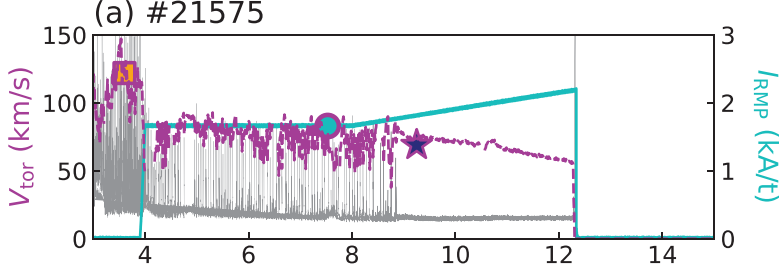

(b) \#21574

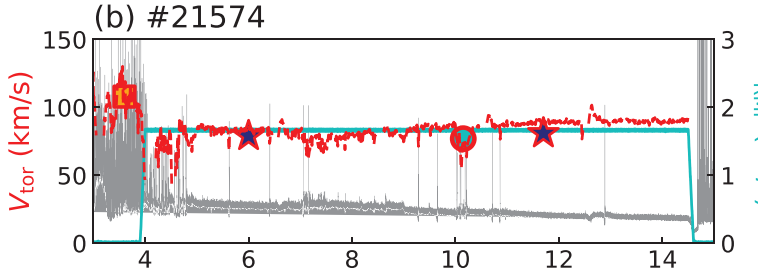

(c) \#21576

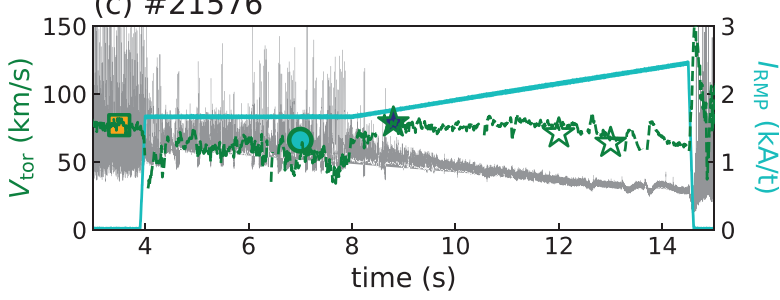

FIG. 7. Time trace of the CES measurement around pedestal top in (a) $\# 21575$ $\left(\nu_{\text {e,ped }}^{*} \sim 0.25\right)$, (b) \#21574 $\left(\nu_{\text {e.ped }}^{*} \sim 0.45\right)$, and (c) \#21576 $\left(\nu_{\text {e, ped }}^{*} \sim 0.75\right)$ with $I_{\text {RMP }}$ (cyan) and $D_{\alpha}$ (gray) signals. $n_{\text {e,ped }}$ and $\nu_{\text {e.ped }}^{*}$ corresponding to each marker can be found in Fig. 6.

$\nu_{\text {e.ped }}^{*}, q_{95}$, and $I_{\mathrm{RMP}}$ (in $\mathrm{kA} /$ turn), are taken into account in LDA after standardization $\left[x_{\text {std }}=\left(x-m_{x}\right) / \sigma_{x}\right.$, where $m_{x}$ and $\sigma_{x}$ are the mean and standard deviation of variable $x$ ]. Since two classes (mitigation and suppression) are considered here, only one eigenvector has a nonzero eigenvalue: $w=0.27 Z\left(n_{\text {e,ped }}\right)-0.02 Z\left(T_{\text {e,ped }}\right)-0.05 Z\left(T_{\mathrm{i}, \text { ped }}\right)$ $-0.63 Z\left(V_{\text {tor,ped }}\right)-0.44 Z\left(\nu_{\text {e.ped }}^{*}\right)-0.13 Z\left(q_{95}\right)-0.57 Z\left(I_{\mathrm{RMP}}\right)$, where $Z\left(x_{\text {std }}\right)$ indicates a unit vector corresponding to a standardized variable $x_{\text {std. }}$. Figure 9 shows data distributions of ELM-crash mitigation and suppression in a new axis named LD1. The horizontal axis is set as $n_{\mathrm{e}, \text { ped }}$ to compare the data distributions in LD1 space with those in $\nu_{\text {e,ped }}^{*}-n_{\text {e,ped }}$ [Fig. 5(a)], $T_{\text {e,ped }}-n_{\text {e,ped }}\left[\right.$ Fig. 5(b)], and $V_{\text {tor,ped }}-n_{\text {e,ped }}$ space [Fig. 6(a)] directly. Although a boundary from LDA (dotted line) does

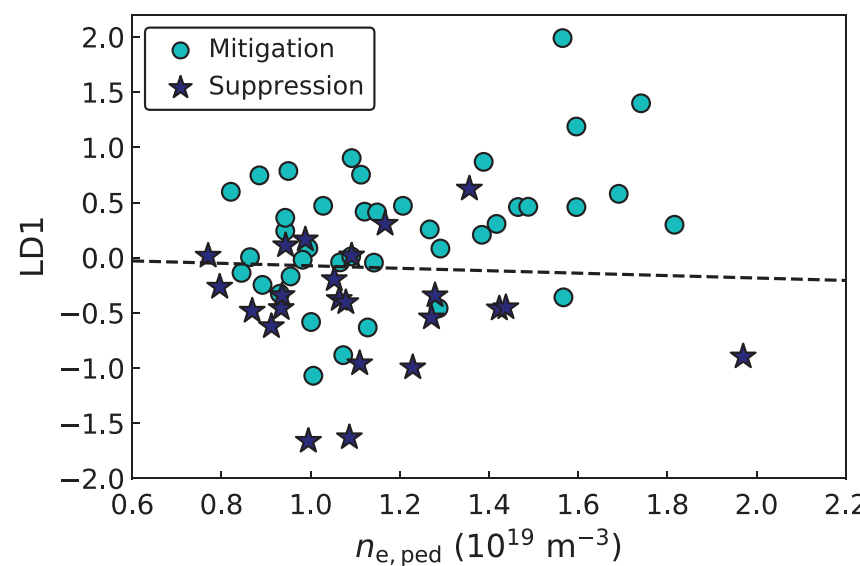

FIG. 9. Data distribution of ELM-crash mitigation (circle) and ELM-crash suppression (pentagram) in LD1 vs electron density space. LD1: parameter space constructed by linear discriminant analysis (LDA). Dotted line: a boundary between two data categories from LDA prediction.

not completely divide the two groups, the separation of two groups is better in LD1 space than in each $\nu_{\text {e,ped }}^{*}, n_{\text {e,ped }}, T_{\text {e,ped}}$, and $V_{\text {tor,ped }}$ parameter space.

Using the Scikit-learn library ${ }^{40}$ for Python programming language, recursive feature elimination $(\mathrm{RFE})^{41}$ is introduced to investigate which variables included in LDA are more relevant to the group separation. RFE evaluates the importance of variables and removes the least important variable recursively. RFE shows that $n_{\mathrm{e} \text {,ped }}, I_{\mathrm{RMP}}$, and $\nu_{\text {e,ped }}^{*}$ are the most important variables, and the next is $q_{95}$ and $V_{\text {tor,ped }}$. Both $n_{\mathrm{e}}$ and $T_{\mathrm{e}}$ are related to $\nu_{\mathrm{e}}^{*}$, but $T_{\mathrm{e}}$ contributes less to the group separation in the current datasets. Although it is not simple to connect the RFE result to the data distribution shown in Figs. 5 and 6, the data distributions in $n_{\text {e,ped }}$ and $I_{\mathrm{RMP}}$ space can explain the RFE result. In $n_{\mathrm{e}, \mathrm{ped}}$ and $I_{\mathrm{RMP}}$ space, the data distributions of the two categories are somewhat distinct. A certain number of mitigation data points are distributed outside the region where the suppression points are clustered: $n_{\text {e,ped }}>1.5 \times 10^{19} \mathrm{~m}^{-3}$ [Figs. 5(a) and 5(b)] and $I_{\mathrm{RMP}}<1.6 \mathrm{kA} / \mathrm{t}$ [Figs. 5(c) and 6(c)].

The LDA and RFE results suggest that it is necessary to consider several parameters together and optimize the weight of the variables to clarify ELM-crash suppression access conditions.
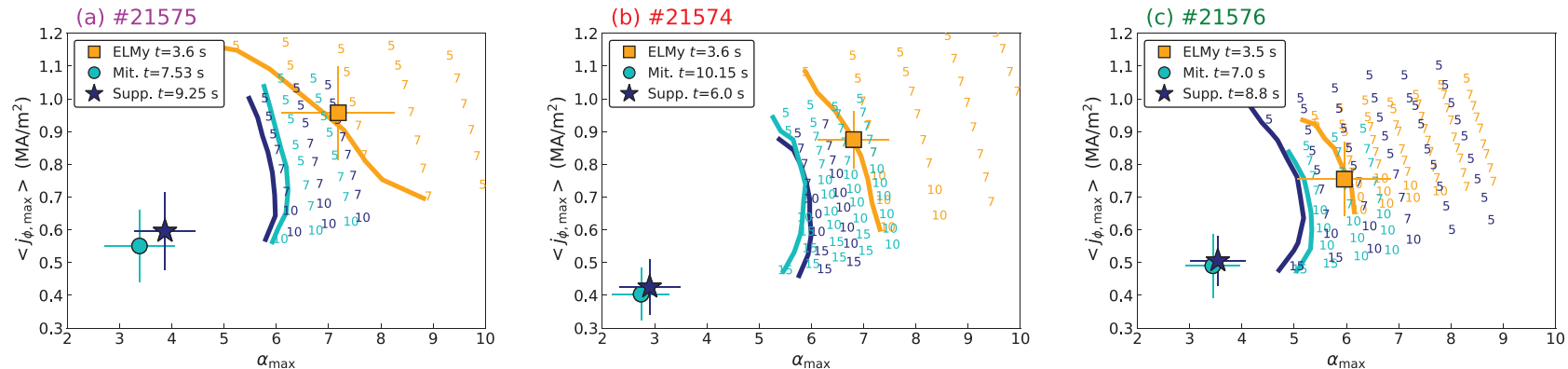

FIG. 8. MISHKA1 ideal linear stability calculation for (a) \#21575 ( $\nu_{\mathrm{e} \text { ed }}^{*} \sim 0.25$ ), (b) \#21574 ( $\left.\nu_{\mathrm{e} \text { ped }}^{*} \sim 0.45\right)$, and (c) \#21576 ( $\left.\nu_{\mathrm{e} \text { ped }}^{*} \sim 0.75\right)$. Solid lines: stability boundary for ELMy (orange), mitigation (cyan), and suppression phases (blue). Integer numbers: toroidal mode number of the most unstable mode. 


\section{SUMMARY}

Based on the high reproducibility of RMP-driven ELM-crash suppression in KSTAR, we established a discharge database to investigate the plasma parameters required for ELM-crash suppression. A total of 28 discharges in the database have a similar plasma shape ( $\delta \sim 0.6)$, and the RMP configuration of all the discharges has been selected as $n=1,+90^{\circ}$ phasing. Among various factors that could be responsible for the ELM-crash suppression, we have explored $\nu_{\mathrm{e}}^{*}$ and $V_{\text {tor }}$ at the pedestal top as key parameters. The edge pedestal parameters of interest are quantified utilizing the modified hyperbolic tangent curve based on time-averaged Thomson scattering and charge exchange spectroscopy data. The ELM-crash suppression points from the discharges in the database are in the range of $0.2<\nu_{\text {e.ped }}^{*}<1.1$ with $Z_{\text {eff }}=2$ assumption and $V_{\text {tor,ped }}>40 \mathrm{~km} / \mathrm{s}$. However, these parameter ranges do not indicate the ELM-crash suppression boundaries or thresholds. The full range of accessible pedestal parameters during the RMP phase in KSTAR, such as $n_{\text {e,ped }}>2.5 \times 10^{19} \mathrm{~m}^{-3}$, is not investigated yet. In the near future, we plan to conduct the experiments in a region of $\nu_{\text {e,ped }}^{*}<0.2$ and $V_{\text {tor,ped }}<40 \mathrm{~km} / \mathrm{s}$, to address the feasibility of the RMP technique in the ITER-relevant conditions $\left(\nu_{\text {e,ped }}^{*} \sim 0.1\right.$ and low-torque low-rotation).

The distributions of ELM-crash suppression are not distinct from those of ELM-crash mitigation in the investigated parameter space. Linear discriminant analysis (LDA) provides a projection vector, consisting of a linear combination of parameters relevant to the ELM-crash suppression, which best separates the two data categories. Recursive feature elimination (RFE) ranks the importance of variables involved in the LDA process; $n_{\mathrm{e}, \text { ped }}$ and $I_{\mathrm{RMP}}$, as well as $\nu_{\text {e.ped }}^{*}$ and $V_{\text {tor,ped, }}$, are critical in the separation of data categories. The LDA and RFE analysis result suggests that several parameters need to be considered to study the access conditions of the ELMcrash suppression.

\section{ACKNOWLEDGMENTS}

The authors thank all the KSTAR team members for their support and Dr. Jong-Kyu Park, in PPPL, for the discussion about the collisionality effect on the ELM-crash suppression conditions. This research was supported by the Korea Ministry of Science and ICT under the NFRI R\&D program (No. NFRI-EN2001-11) and by the National Research Foundation (NRF) of Korea under Grant No. NRF-2019M1A7A1A03088443.

\section{DATA AVAILABILITY}

The data that support the findings of this study are available from the corresponding author upon reasonable request.

\section{REFERENCES}

${ }^{1}$ A. Loarte, G. Huijsmans, S. Futatani, L. Baylor, T. Evans, D. Orlov, O. Schmitz, M. Becoulet, P. Cahyna, Y. Gribov et al., Nucl. Fusion 54, 033007 (2014).

${ }^{2}$ T. E. Evans, R. A. Moyer, P. R. Thomas, J. G. Watkins, T. H. Osborne, J. A. Boedo, E. J. Doyle, M. E. Fenstermacher, K. H. Finken, R. J. Groebner, M. Groth, J. H. Harris, R. J. La Haye, C. J. Lasnier, S. Masuzaki, N. Ohyabu, D. G. Pretty, T. L. Rhodes, H. Reimerdes, D. L. Rudakov, M. J. Schaffer, G. Wang, and L. Zeng, Phys. Rev. Lett. 92, 235003 (2004).

${ }^{3}$ Y. Jeon, J.-K. Park, S. Yoon, W. Ko, S. Lee, K. Lee, G. Yun, Y. Nam, W. Kim, J.-G. Kwak et al., Phys. Rev. Lett. 109, 035004 (2012).
${ }^{4}$ J. Lee, G. S. Yun, M. J. Choi, J.-M. Kwon, Y.-M. Jeon, W. Lee, N. C. Luhmann, Jr., and H. K. Park, Phys. Rev. Lett. 117, 075001 (2016).

${ }^{5}$ Y. In, J.-K. Park, Y. Jeon, J. Kim, G. Park, J.-W. Ahn, A. Loarte, W. Ko, H. Lee, J. Yoo et al., Nucl. Fusion 57, 116054 (2017).

${ }^{6}$ Y. In, Y. Jeon, J.-K. Park, A. Loarte, J.-W. Ahn, J. Lee, H. Lee, G. Park, K. Kim, H. Kim et al., Nucl. Fusion 59, 056009 (2019).

${ }^{7}$ J. Lee, Y. Jeon, Y. In, G. Park, G. Yun, W. Lee, M. Kim, J. Lee, W. Ko, H. K. Park et al., Nucl. Fusion 59, 066033 (2019).

${ }^{8}$ W. Suttrop, T. Eich, J. Fuchs, S. Günter, A. Janzer, A. Herrmann, A. Kallenbach, P. Lang, T. Lunt et al., Phys. Rev. Lett. 106, 225004 (2011).

${ }^{9}$ W. Suttrop, A. Kirk, V. Bobkov, M. Cavedon, M. Dunne, R. McDermott, H. Meyer, R. Nazikian, C. Paz-Soldan, D. A. Ryan et al., Nucl. Fusion 58, 096031 (2018).

${ }^{10}$ A. Kirk, W. Suttrop, I. Chapman, Y. Liu, R. Scannell, A. Thornton, L. B. Orte, P. Cahyna, T. Eich, R. Fischer et al., Nucl. Fusion 55, 043011 (2015).

${ }^{11}$ Y. Liang, P. Lomas, I. Nunes, M. Gryaznevich, M. Beurskens, S. Brezinsek, J. Coenen, P. Denner, T. Eich, L. Frassinetti et al., Nucl. Fusion 53, 073036 (2013).

${ }^{12}$ T. Petrie, T. Evans, N. Brooks, M. Fenstermacher, J. Ferron, C. Holcomb, B. Hudson, A. Hyatt, T. Luce, C. Lasnier et al., Nucl. Fusion 51, 073003 (2011).

${ }^{13}$ C. Paz-Soldan, R. Nazikian, L. Cui, B. Lyons, D. Orlov, A. Kirk, N. Logan, T. Osborne, W. Suttrop, and D. Weisberg, Nucl. Fusion 59, 056012 (2019).

${ }^{14}$ T. Evans, A. Loarte, D. Orlov, B. Grierson, M. Knölker, B. Lyons, L. Cui, P. Gohil, R. Groebner, R. Moyer et al., Nucl. Fusion 57, 086016 (2017).

${ }^{15}$ R. Moyer, T. Evans, T. Osborne, P. Thomas, M. Becoulet, J. Harris, K.-H. Finken, J. Boedo, E. Doyle, M. Fenstermacher et al., Phys. Plasmas 12, 056119 (2005).

${ }^{16}$ R. A. Moyer, C. Paz-Soldan, R. Nazikian, D. M. Orlov, N. Ferraro, B. A. Grierson, M. Knölker, B. Lyons, G. R. McKee, T. H. Osborne et al., Phys. Plasmas 24, 102501 (2017).

${ }^{17}$ H. Han, Y. Jeon, S. Hahn, H. Ahn, J. Bak, Y. In, J. Kim, M. Woo, H. Kim, J. Jin et al., Fusion Eng. Des. 108, 60 (2016).

${ }^{18}$ T. Evans, D. Orlov, A. Wingen, W. Wu, A. Loarte, T. Casper, O. Schmitz, G. Saibene, M. Schaffer, and E. Daly, Nucl. Fusion 53, 093029 (2013).

${ }^{19}$ Y. In, A. Loarte, H. Lee, K. Kim, Y. Jeon, J.-K. Park, J.-W. Ahn, G. Park, M. Kim, H. Park et al., Nucl. Fusion 59, 126045 (2019).

${ }^{20}$ J.-K. Park, Y. Jeon, Y. In, J.-W. Ahn, R. Nazikian, G. Park, J. Kim, H. Lee, W. Ko, H.-S. Kim et al., Nat. Phys. 14, 1223 (2018).

${ }^{21}$ J. Lee, S. Oh, and H. Wi, Rev. Sci. Instrum. 81, 10D528 (2010).

${ }^{22}$ W.-H. Ko, H. Lee, D. Seo, and M. Kwon, Rev. Sci. Instrum. 81, 10 D740 (2010).

${ }^{23}$ A. Kallenbach, R. Dux, J. Gafert, G. Haas, L. Horton, M. Jakobi, B. Kurzan, H. Müller, R. Neu, J. Neuhauser et al., Nucl. Fusion 43, 573 (2003).

${ }^{24}$ L. Lao, H. S. John, R. Stambaugh, A. Kellman, and W. Pfeiffer, Nucl. Fusion 25, 1611 (1985).

${ }^{25}$ Y. Nam and K. Lee, Rev. Sci. Instrum. 79, 10E705 (2008).

${ }^{26}$ O. Sauter, C. Angioni, and Y. Lin-Liu, Phys. Plasmas 6, 2834 (1999).

${ }^{27}$ Q. Hu, R. Nazikian, B. Grierson, N. Logan, J.-K. Park, C. Paz-Soldan, and Q. Yu, Phys. Plasmas 26, 120702 (2019).

${ }^{28}$ Q. Hu, R. Nazikian, B. A. Grierson, N. Logan, C. Paz-Soldan, and Q. Yu, Nucl. Fusion 60, 076001 (2020).

${ }^{29}$ M. Greenwald, J. Terry, S. Wolfe, S. Ejima, M. Bell, S. Kaye, and G. Neilson, Nucl. Fusion 28, 2199 (1988).

${ }^{30}$ J. Ahn, H.-S. Kim, Y. Park, L. Terzolo, W. Ko, J.-K. Park, A. England, S. Yoon, Y. Jeon, S. Sabbagh et al., Nucl. Fusion 52, 114001 (2012).

${ }^{31}$ K. Kim, Y. Jeon, J.-K. Park, W. Ko, Y. In, W. Choe, J. Kim, S. Lee, S. Yoon, J. Kwak et al., Nucl. Fusion 57, 036014 (2017).

${ }^{32}$ A. Mikhailovskii, G. Huysmans, W. Kerner, and S. Sharapov, Plasma Phys. Rep. 23, 844 (1997).

${ }^{33}$ R. Miller, M.-S. Chu, J. Greene, Y. Lin-Liu, and R. Waltz, Phys. Plasmas 5, 973 (1998).

${ }^{34}$ P. Snyder, R. Groebner, J. Hughes, T. Osborne, M. Beurskens, A. Leonard, H. Wilson, and X. Xu, Nucl. Fusion 51, 103016 (2011). 
${ }^{35}$ M. Willensdorfer, T. Cote, C. Hegna, W. Suttrop, H. Zohm, M. Dunne, E. Strumberger, G. Birkenmeier, S. S. Denk, F. Mink et al., Phys. Rev. Lett. 119, 085002 (2017).

${ }^{36}$ M. Willensdorfer, T. Cote, M. Griener, D. Ryan, E. Strumberger, W. Suttrop, N. Wang, M. Cavedon, S. Denk, M. Dunne et al., Plasma Phys. Controlled Fusion 61, 014019 (2019).

${ }^{37}$ R. A. Fisher, Ann. Eugenics 7, 179 (1936).
${ }^{38}$ S. Raschka, Python Machine Learning (Packt Publishing Ltd., 2015).

${ }^{39}$ R. O. Duda, P. E. Hart, and D. G. Stork, Pattern Classification (John Wiley and Sons, 2001).

${ }^{40}$ F. Pedregosa, G. Varoquaux, A. Gramfort, V. Michel, B. Thirion, O. Grisel, M. Blondel, P. Prettenhofer, R. Weiss, V. Dubourg et al., J. Mach. Learn. Res. 12, 2825 (2011).

${ }^{41}$ I. Guyon, J. Weston, S. Barnhill, and V. Vapnik, Mach. Learn. 46, 389 (2002). 